Int. J. Odontostomat.,

7(2):221-224, 2013.

\title{
Displasia Fibrosa Monostótica Craneofacial: ¿Cómo Debe Ser el Diagnóstico?
}

\author{
Monostotic Craniofacial Fibrous Dysplasia: How Should it be Diagnosed?
}

\author{
Nilton Alves
}

ALVES, N. Displasia fibrosa monostótica craneofacial: ¿cómo debe ser el diagnóstico? Int. J. Odontostomat., 7(2):221224, 2013.

RESUMEN: La displasia fibrosa (DF) es una patología benigna rara, generalmente asintomática, que afecta el tejido óseo. Debido al remplazo gradual del tejido óseo por tejido conectivo amorfo se pueden producir alteraciones óseas estéticas y funcionales. En este artículo se realiza una revisión de la literatura sobre la DF y se relata un caso clínico de una mujer de 49 años de edad que presenta deformidad facial debida al crecimiento lento y progresivo del maxilar derecho. Con esto pretendemos ofrecer una secuencia diagnóstica para establecer el diagnóstico definitivo de la DF monostótica, brindando los principios básicos para el manejo adecuado de los pacientes que sufren de esta enfermedad.

PALABRAS CLAVE: displasia fibrosa monostótica, anomalías maxilomandibulares, tomografía computarizada cone-beam.

\section{INTRODUCCIÓN}

La displasia fibrosa (DF) es una enfermedad rara, que afecta el tejido óseo. Se trata de una patología benigna, caracterizada por el remplazo de tejido óseo por tejido conectivo amorfo, lo que puede resultar en alteraciones estéticas y funcionales. Suele ser asintomática, aunque a veces por su localización, puede determinar múltiples síntomas. La DF puede afectar un hueso (monostótica) o varios huesos (poliostótica), siendo más común su manifestación en la infancia o al inicio de la adolescencia (Lichtenstein \& Jaffe, 1942). La DF poliostótica relacionase con puberdad temprana y áreas de pigmentación cutánea (McCune, 1936; Albright et al., 1937). Su etiología es debida a un desequilibrio en la función de las células osteogénicas, las lesiones aparecen por una mutación que activa la subunidad a, que codifica la proteína $G$ en las células osteoblásticas y produce una matriz ósea fibrosa desorganizada (Cohen \& Howell, 1999). Otros autores plantean que puede ser una reacción anormal del hueso a un episodio traumático local o un desorden endocrino que se manifiesta como una condición ósea focal (Regezi \& Sciubba, 2000).

La DF se caracteriza por un aumento lento y deformante del hueso comprometido, siendo que en la variedad más frecuente, que es la monostótica, son los maxilares los huesos más afectados, seguidos por las costillas y el fémur. Otros huesos del cráneo pueden estar comprometidos, como el temporal, el etmoides y el esfenoides, esta variedad es conocida como displasia fibrosa craneofacial (DFCF) (Regezi \& Sciubba; Ricalde \& Horswell, 2001). Radiográficamente las lesiones de DF se caracterizan por un aspecto de vidrio deslustrado de límites mal definidos, debido a la mezcla de elementos óseos y fibrosos. Puede ser unilocular o multilocular y muchas veces está asociada con expansión de la cortical ósea. El mejor método para identificar la DFCF es la tomografía computarizada ya que permite determinar la localización y extensión de la lesión, auxilia en la planificación del tratamiento y en el procedimiento quirúrgico (Regezi \& Sciubba).

\section{REPORTE DEL CASO}

Paciente de sexo femenino, caucasiano, 49 años de edad, compareció a la consulta debido a un aumento de volumen lento e indoloro del maxilar de- 
recho. En la anamnesis, la paciente refirió parestesia en la región comprometida.

Al examen físico se observó asimetría facial, por el aumento de tamaño del maxilar derecho, sin signos inflamatorios.

Se realizó examen de tomografía computarizada cone-beam (TCCB), que evidenció imagen compatible con displasia fibrosa. La lesión comprometía seno maxilar derecho (Figs. 1, 2 y 3).

Fue realizada la biopsia incisional para examen microscópico de la lesión y confirmación de la hipótesis de diagnóstico que reveló el resultado de displasia fibrosa, ante lo cual se realizó una gammagrafía ósea y un estudio endocrinológico para descartar el Síndrome de Mc Cune-Albright (formas poliostóticas). El resultado de todas las exploraciones fue negativo, por lo que se diagnosticó como displasia fibrosa monostótica del seno maxilar derecho.

Basados en la extensión de la lesión, la paciente ha sido sometida a procedimiento quirúrgico con realización de osteotomía y plastia ósea. Además, ha sido orientada a retornar para el control de recurrencias.

\section{DISCUSIÓN}

La DF es una enfermedad ósea benigna que manifestase con mayor frecuencia en los niños y adolescentes, aunque pueda ocurrir en los adultos como en el caso aquí presentado.
Se han descrito casos de DF asociados con hipertiroidismo, diabetes insípida, hipercortisolismo, hiperprolactinemia y acromegalia (Yavuzer et al., 1999).

La DF puede afectar un hueso (monostótica), varios huesos (poliostótica) y puede acompañar al Síndrome de Mc Cune-Albright que consiste, como describió Albright et al., en lesiones de osteítis fibrosa diseminada, áreas de pigmentación cutánea (manchas "café con leche") y alteraciones endocrinas como pubertad precoz (Carreto Sanchez et al.,1997; Carmen Sampériz et al., 2000).

En el caso aquí relatado, la paciente, con 49 años de edad, compareció a la consulta debido a un aumento de volumen lento e indoloro del maxilar derecho, se realizó examen de tomografía computarizada cone-beam, que evidenció imagen compatible con displasia fibrosa. La forma monostótica, que es la que se detecta más tardíamente, está caracterizada por la aparición aislada de las lesiones óseas. Ante la aparición de las mismas es necesario descartar el síndrome mediante estudio clínico y endocrinológico y gammagrafía ósea en busca de otras lesiones en sitios insospechados (Carmen Sampériz et al.).

Cuando la lesión afecta la estética facial y la función, como en nuestro caso, el tratamiento quirúrgico debe ser indicado. En caso contrario, debe ser acompañada clínicamente y a través del control imagenológico. Así, hay que individualizar el tratamiento para cada caso y en muchas ocasiones es obligatorio un seguimiento de estos pacientes, una vez que hay un gran porcentaje de recidivas. Según algunos autores las recidivas llegan a un 10 a $25 \%$ de los ca-

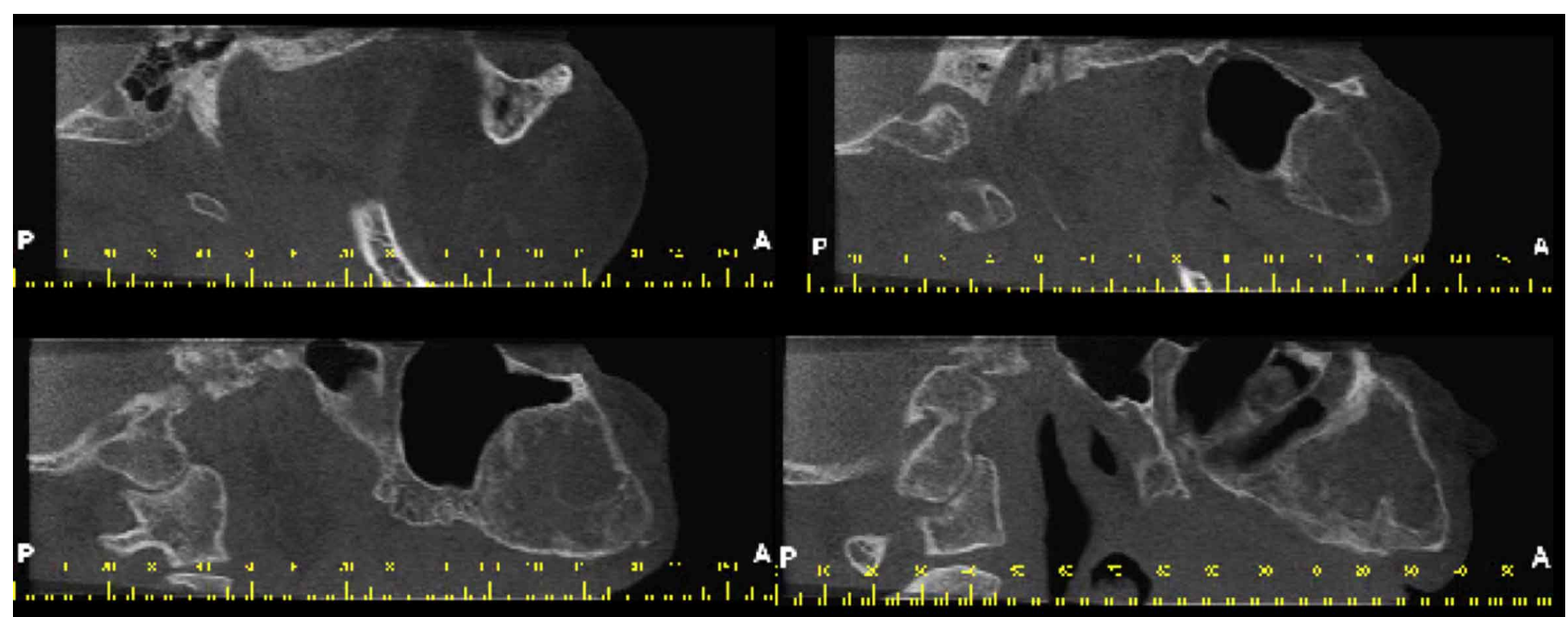

Fig. 1. Cortes sagitales de TCCB, mostrando imágenes compatibles con DF en región de seno maxilar derecho. 


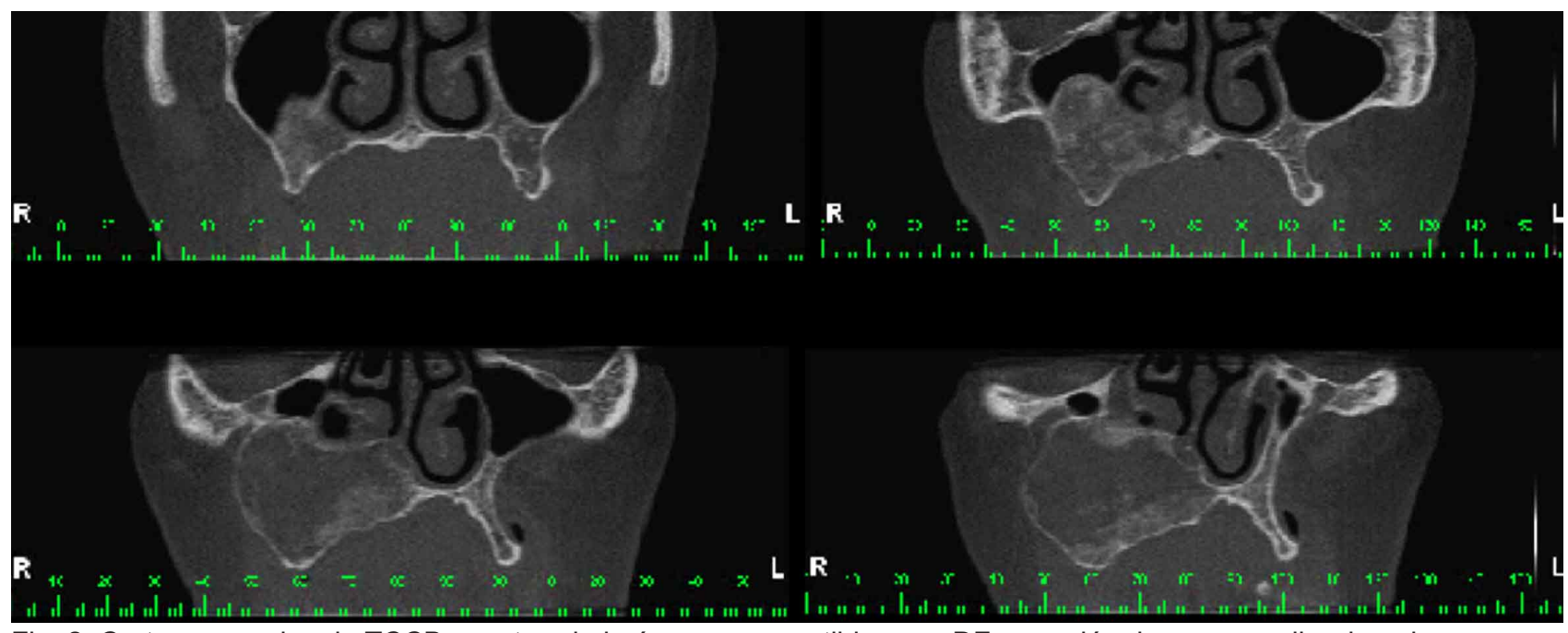

Fig. 2. Cortes coronales de TCCB, mostrando imágenes compatibles con DF en región de seno maxilar derecho.

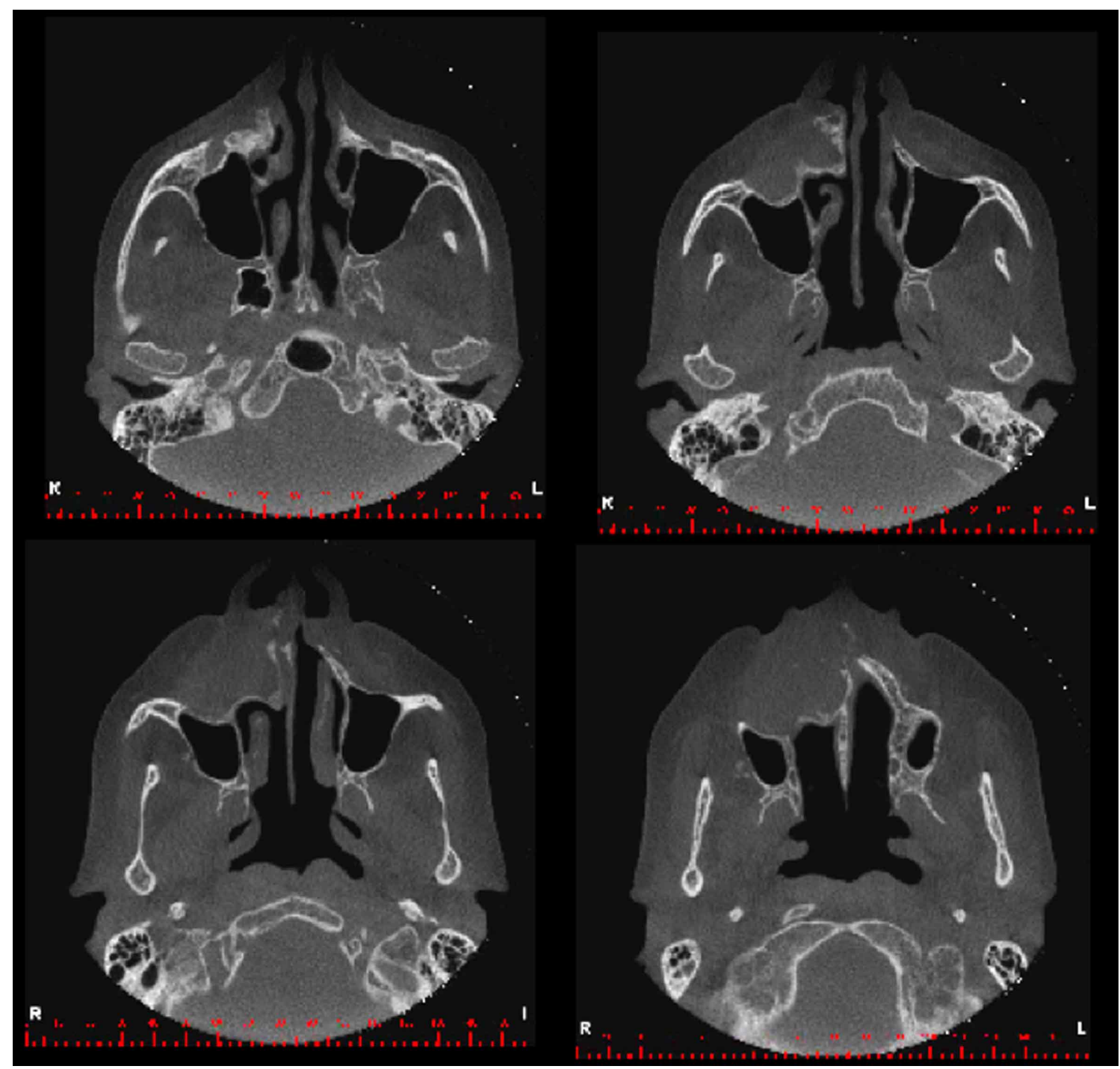

Fig 3. Cortes axiales de TCCB, mostrando imágenes compatibles con DF en región de seno maxilar derecho. 
sos (Bibby \& McFadzean, 1994; Simovic et al., 1996; Carreto Sanchez et al.; Carmen Sampériz et al.).

Por lo tanto, para un correcto diagnóstico es muy importante un examen imagenológico, un análisis histopatológico y ante la aparición de una DF en el área maxilofacial, descartar nuevas localizaciones y el Síndrome de Mc Cune-Albright mediante estudio endocrinológico y gammagrafía ósea que es la forma más rápida para buscar otras lesiones en sitios insospechados. El examen imagenológico de elección es la tomografía computarizada cone-beam ya que ofrece una visión más amplia de la extensión y localización de la lesión, auxiliando en la planificación del procedimiento quirúrgico.

ALVES, N. Monostotic craniofacial fibrous dysplasia: how should it be diagnosed? Int. J. Odontostomat., 7(2):221-224, 2013.

ABSTRACT: Fibrous dysplasia (FD) is a rare benign pathology, generally asymptomatic affecting the bone tissue. Due to the gradual replacement of the bone tissue by amorphous connective tissue, aesthetic and functional bone alterations can occur. A review of the literature on FD is made and we report a clinical case of a 49-year-old woman presenting facial deformity as a result of the slow and progressive growth of the right maxillar. With this paper, we intend to offer a diagnostic sequence to establish the definitive diagnosis of monostotic FD, providing the basic principles for the proper management of patients suffering from this disease.

KEY WORDS: monostotic fibrous dysplasia, maxillomandibular abnormalities, cone-beam computed tomography.

\section{REFERENCIAS BIBLIOGRÁFICAS}

Albright, F.; Butler, A. M.; Hampton, A. O. \& Smith, P. Syndrome characterized by osteitis fibrosa disseminata, areas of pigmentation and endocrine dysfunction, with precocious puberty in females - Report of five cases. $N$. Engl. J. Med., 216(17):727-46, 1937.

Bibby, K. \& McFadzean, R. Fibrous dysplasia of the orbit. Br. J. Ophtalmol., 78(4):266-70, 1994.

Carmen Sampériz, L.; Damborenea Tejada, J.; Fernández Liesa, R.; Llorente Arenas, E. M.; Marín Garrido, C.; Naya Gálvez, M. J.; et al. Displasia fibrosa craneofacial. A propósito de dos casos. ORL Dips., 27(2):74-8, 2000.

Carreto Sanchez, J.; Balderrama Caballero, D. H. \& Relea Calatayud, M. F. Displasia fibrosa monostótica del maxilar superior. Presentación de un caso clínico. Acta Otorrinolaringol. Esp., 48:579-82, 1997.

Cohen, M. M. Jr. \& Howell, R. E. Etiology of fibrous dysplasia and McCune-Albright syndrome. Int. J. Oral Maxilofac. Surg., 28(5):366-71, 1999.

Lichtenstein, L. \& Jaffe, H. L. Fibrous dysplasia of bone: a condition affecting one, several or many bones, the graver cases of which may present abnormal pigmentation of skin, premature sexual development, hyperthyroidism or still other extra skeletal abnormalities. Arch. Pathol., 33:777-81, 1942.

McCune, D. J. Osteitis fibrosa cystica. The case of a nine year old girl who also exhibits precocious puberty, multiple pigmentation of the skin and hyperthyroidism. Am. J. Dis. Child., 52:743-7, 1936.

Regezi, J. A. \& Sciubba, J. J. Patologia bucal: correlações clinicopatológicas. $3^{a}$ ed. Philadelphia, GuanabaraKoogan, 2000. pp.324-6.

Ricalde, P. \& Horswell, B. B. Craniofacial fibrous dysplasia of the fronto-orbital region: A case series and literature review. J. Oral Maxillofac. Surg., 59(2):157-68, 2001.

Simovic, S.; Klapan, I.; Bumber, Z. \& Bura, M. Fibrous dysplasia in paranasal cavities. ORL J. Otorhinolaryngol. Relat. Spec., 58(1):55-8, 1996.

Yavuzer, R.; Khilnani, R.; Jackson, I. T. \& Audet, B. A case of atypical McCune-Albright syndrome requiring optic nerve decompression. Ann. Plast. Surg., 43(4):430-5, 1999.

Dirección para correspondencia

Prof. Dr. Nilton Alves

Departmento de Odontología Integral Adultos

Universidad de La Frontera

Claro Solar No 115, Piso 40, Temuco

CHILE

Email: niltonnalves@yahoo.com.br

Recibido : 31-08-2012

Aceptado: 22-03-2013 